\title{
Die Ökonomisierung der Medizin: Ist die Heilkunst ein Business?
}

\author{
The Economization of Medicine: Is Medicine a Business?
}

\author{
Thomas F. Lüscher ${ }^{1,2,3}$ \\ ${ }^{1}$ Royal Brompton \& Harefield Hospital and Imperial College, London, United Kingdom \\ ${ }^{2}$ Center for Molecular Cardiology, Universität Zürich \\ ${ }^{3}$ Foundation for Cardiovascular Research - Zurich Heart House, Zürich
}

\section{Die Frage}

Eine unvorhergesehene Einladung wie die, einen Beitrag zur Ökonomisierung der Medizin zu verfassen, bedarf der Klärung: Was ist Ökonomisierung? Wenn wir unseren ständigen Ratgeber Wikipedia fragen, so lesen wir, dass der Begriff die Ausbreitung des Marktes bzw. seiner Ordnungsprinzipien und Prioritäten auf Bereiche beschreibt, in denen ökonomische Überlegungen in der Vergangenheit eine eher untergeordnete Rolle spielten bzw. die solidarisch oder privat organisiert waren. Nun gut: Was hiesse Ökonomisierung in der Medizin? Gewiss, das Lindern und Heilen war zunächst ein solidarisch betriebenes Geschäft; Gewinn war nicht vorgesehen. Jedoch: Dass Geld in der Medizin heute eine «dominante Rolle spielt, kann kaum einem Zeitungsleser entgehen. Die Ordnungsprinzipien des Markts in der kapitalistischen Gesellschaft wie der unseren fordern Konkurrenz, Wachstum und Gewinnstreben - dem war nicht immer so.

\section{Die Anfänge}

In der Bibel, so lesen wir im Lukas-Evangelium, ist die Rede von einem Juden, der unterwegs nach Jericho überfallen und fast erschlagen wurde. Ein Samariter - ein Vertreter eines Stammes also, der mit Judäa über Jahre im Streit lag -, wandte sich ihm zu, goss Öl und Wein über seine Wunden, hob ihn auf sein Reittier, brachte ihn zu einer Herberge, und pflegte ihn - im Buch der Bücher wird der Samariter zum Urbild des Arztes [1]. Obgleich der Samariter über Jahre die Schweizer Hunderternote zierte (Abb. 1), ist nichts von einem Honorar für seine Taten bekannt. Gewiss, seine Massnahmen liegen weit hinter den heutigen zurück; doch es ist nichts unhinterfragbar gut, denn ein guter Wille, wie Kant Jahrhunderte später meinte [2].

Im Altertum, als die Heilkunst wenig zu bieten hatte, mahnte Hippokrates zur Vorsicht: Primum nil nocere [3] -, noch ging es vordringlich um Beistand bei Schmerz und

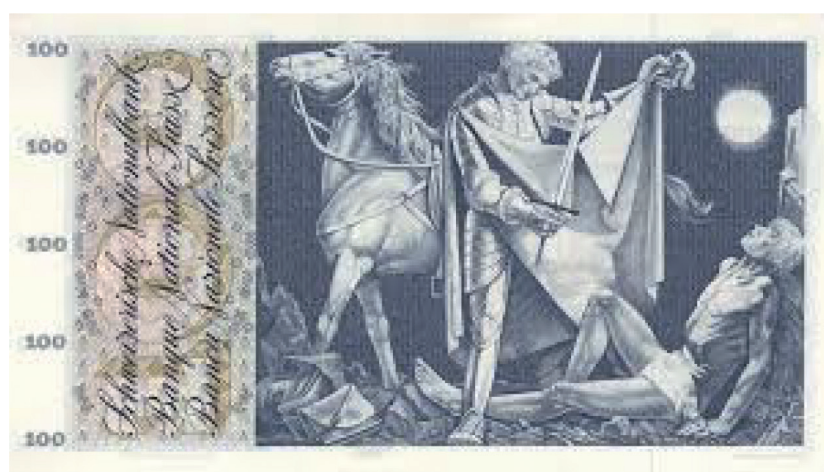

Abbildung 1. Der gute Samariter auf der alten Schweizer Hunderternote.

Leid, mehr liess sich kaum versprechen. Ja, Weitergehendes barg die Gefahr, zu schaden. Dann machte die Heilkunst über Hunderte von Jahren ihrem Namen vorerst zaghaft, dann beeindruckend alle Ehre: Nach dem Lindern kam das Behandeln. Beschwerden und Behinderungen liessen sich zunehmend angehen, die Medizin gewann Respekt. Doch Heilen gelang nur selten, vielleicht seit der Entdeckung von Penicillin und Streptomycin bei Infektionen, dann bei Verletzungen und Unfällen. Mit der Impfung kam die Vorsorge zu ihrem Recht. Sie bewahrte uns vor den Geisseln der Menschheit, zunächst dank Jenners heroischer Tat [4] vor den Pocken, dann vor Polio, Masern, Diphtherie und Rubeolen - kurz: eine einmalige Erfolgsgeschichte.

\section{Medical Nemesis}

Unterstützt von der sich ausbreitenden Hygiene gewährte uns dies eine unerwartete Langlebigkeit. Doch blieb sie nicht ohne Folgen: Ein langes Leben war in der Evolution nicht vorgesehen. Heute, da wir uns der natürlichen Selektion enthoben haben, plagen uns chronische Erkrankungen, abgenutzte Gelenke, die das Gehen zur Tortur werden lassen, schwache Herzen, die länger als vorgesehen 
schlagen und uns schwer atmen lassen. Dann Gene, die von der Evolution nicht entsorgt wurden und sich im Alter bemerkbar machen: die hohen Lipidwerte des Menschen, die zu Arteriosklerose, Infarkt und Herztod führen. Ein auf Mangel programmierter Metabolismus gerät mit der allgegenwärtigen Verfügbarkeit von Snacks, Süssigkeiten, Softdrinks und kalorienreicher Nahrung ausser Rand und Band; es kommt zu Übergewicht, Diabetes und ihren Folgen. Dann wachsen mit zunehmendem Alter Tumoren, die behandelt werden wollen. All dies lässt sich angehen, aber selten heilen - eine ausgeklügelte Diagnostik, teure Eingriffe und jahrzehntelange Behandlungen sind die Folgen. Dass dies die Kosten in die Höhe treibt, erstaunt wohl keinen.

\section{Professionalisierung}

Seit dem barmherzigen Samariter gehörte Selbstlosigkeit zum Arzt und Heiler. Ja, von einem guten Arzt wird erwartet, dass er als Anwalt des Patienten wirkt, nur das Beste und nicht sein Honorar im Auge hat und nur das Nötigste und nicht das Rentabelste verschreibt. Gewiss, dass auch ein Arzt seine Familie zu ernähren hat, wurde klaglos hingenommen, seine Einkünfte schienen verdient. Respekt und Ansehen, deren er sich weiterhin erfreut, schöpften sich aus seiner Zuwendung, seiner Verfügbarkeit und nicht aus seinem Einkommen.

Was für eine gute Ärztin/einen guten Arzt ausser Zuwendung nötig ist, hat sich verändert: War Wissen immer wichtig, so ist die «Knowledge Base» über alle Massen gewachsen - ein Einzelner kann die Medizin nicht mehr überschauen, geschweige denn beherrschen. Spezialisierung und Arbeitsteilung hat die Medizin ergriffen und liess das Personal in alle Höhen wachsen. Die anhaltende Einschränkung der Arbeitszeit in einer sozial geprägten Marktwirtschaft und Teilzeitanstellungen tat das Ihre [5] die Betriebskosten mussten steigen.

Dann die Gerätschaft: Genügte einst das Stethoskop [6] und «Tender Loving Care», so braucht es nun Elektrokardiogramm, Ultraschall, eine Unzahl von Laborbefunden, Computertomografie und Magnetresonanz und vieles mehr. Die therapeutischen Möglichkeiten mit Kathetertechnik, Chirurgie und ständig teureren Medikamenten haben sich beeindruckend erweitert [7]. Ja, In den vergangenen hundert Jahren wurde in der Medizin mehr erreicht als in der gesamten Menschheitsgeschichte davor - auch dies ist nicht ohne Kosten zu haben.

Mit der Technisierung der Medizin wurden auch Ärzte zu Grossverdienern: Zum Ansehen kam das Geld. Und nicht unberechtigt: Wieso sollte ein genialer Neurochirurg wie Mahmut Gazi Yasargil weniger einstecken als ein Direktor einer Bank? Wieso sollte sich Andreas Grüntzig, der die Kardiologie revolutionierte [8], mit einem bescheidenen Salär begnügen? Auch der Allgemeinpraktiker möchte für seinen unermüdlichen Einsatz, seine Dienste und sein Können angemessen vergütet werden.

\section{Die Ankunft der Manager}

Als Folge dieser Kostenspirale begannen die Manager die Krankenhäuser zu übernehmen und private Institutionen, und bald auch öffentliche Krankenhäuser, zu Profitzentren auszubauen - alles wurde auf Wachstum und Gewinn getrimmt. Klinikmanager stehen seither Chefärzten zur Seite und berechnen emsig, was welche Kosten und welches Einkommen schafft. Seither verdrängen glanzvolle Prospekte wissenschaftliche Dokumentationen, der akademische Geist musste dem Businessplan weichen [9]. Nun lesen wir im Tram, im Flugzeug und in der Zeitung von der Kompetenz medizinischer Zentren und ihrer Helden, erfahren in Fernsehspots, wo man sich im Notfall hinwenden soll - kurz, «Branding» und «Public Relations» sind angesagt: Was rentiert, zählt. Und gewiss: Warum soll, was die Wirtschaft erstritt, nicht für die Heilkunst gelten?

Damit ist die Heilkunst nicht, was sie einmal war: Wer Rendite will, schielt auf das Rentable. Profizentren behandeln Herz- und orthopädische Leiden, bieten Eingriffe und Onkologika für bisher unbehandelbare Tumoren denn dies schafft Gewinne. Wachstum lässt sich in einem gesättigten Gesundheitsmarkt wie dem schweizerischen nur durch Ausweitung der Indikationen, durch Verdrängung der Mitbewerber und/oder neue Eingriffe erreichen. Ja, die Kantonalisierung und die Erwartung der Patienten hat zu einem unsinnigen Ausbau von Zentren geführt [10]. Die Schweiz weist nicht ohne Stolz vier- bis fünfmal mehr Herzzentren aus als Holland oder Dänemark. Die Ärztin/ der Arzt hat sich danach zu richten: Die Diagnostik muss optimiert, ihr Einsatz erweitert, und unklare Befunde müssen weiterverfolgt werden. Eingriffe haben möglichst früh und bevorzugt bei Privatpatienten zu erfolgen. Kein Wunder, dass die Kosten laufend steigen.

\section{Rousseau revisited}

Gereicht diese Entwicklung der Medizin zum Guten oder geriet sie auf Abwege, die es zu bekämpfen gilt? Was Rousseau im Jahre 1749 in seinem berühmten Essay mit Bezug auf die kulturelle Entwicklung des Menschen in Frage stellte [11], steht nun in der Medizin zur Diskussion: Hat die Ökonomisierung zur Veredelung oder zum Verderbnis der Sitten in der Medizin beigetragen?

Ohne Zweifel haben die beeindruckenden Möglichkeiten heutiger Samariter die Kosten zwingend steigen lassen; kein Fortschritt ist ohne Kosten zu haben. Was heute Linderung verspricht, wirksam behandelt, gar geheilt werden kann, ist nur mit steigendem finanziellem Einsatz $\mathrm{zu}$ haben: «There is no free lunch!» Alles andere wäre Augenwischerei. Die Ökonomisierung ist Folge und Antrieb des laufenden Fortschritts, und der ist gewollt; niemand würde ein Krankenhaus der 1950er Jahre besuchen wollen [11]. 
Auch Ärzte haben ihre Ansprüche angepasst. Die Aussichten auf einen satten Gewinn lässt auch die Vertreter der Heilkunst nicht kalt. Als Folge treten in einem Gesundheitssystem, das solidarisch finanziert werden muss, die Politiker und in ihrem Windschatten die Beamten auf den Plan. Auch hier gilt: Wer bezahlt, befiehlt! Wo die Mittel des Staates sich beschränken, greifen die Betriebswirtschaftler und Manager nach der Macht. Alles folgt einer politischen und ökonomischen Logik - dennoch stellt sich die Frage nach Wert und Mass.

\section{Segen des Wettbewerbs}

Konkurrenz steigert die Qualität von Dienstleistungen und Waren, und dies gilt auch in der Medizin. Die Sattheit staatlicher Institutionen, welche lange Zeit ein Monopol innehatten und sich nicht um die Zufriedenheit ihrer Kunden zu kümmern brauchten, hat die Qualität ihrer Dienstleistungen nicht gefördert. Dieser Missstand hat den Privatskrankenhäusern den Markt eröffnet. Die Freundlichkeit der Disponenten und Stationsärzte, die Eilfertigkeit der Planer und Sekretäre sowie der Service der Ärztinnen und Ärzte haben sich mit steigender Konkurrenz mit Recht verbessert. Während sich die alten Chefs noch Ausfälle gegen Zuweiser und Kollegen leisten konnten, gilt heute das Prinzip «Der Kunde ist König!» und alle wollen es so.

Hat aber diese Entwicklung auch die Medizin selbst verbessert? Zu betrachten gilt es nicht nur die Eilfertigkeit und Freundlichkeit der Telefonisten, nicht die Verfügbarkeit und Zugänglichkeit der Ärzte, auch nicht die Einrichtung der Krankenzimmer und die Leistungen der Hotellerie, sondern die medizinische Seite der Medaille. Gewiss, die forschende Industrie und ihre Produkte wäre ohne Markt und Konkurrenz nicht zu denken. Ja, ohne Gewinn keine Investment und ohne Einsatz kein registriertes Produkt. Keine Staatswirtschaft hätte hervorgebracht, was wir heute haben.

Wird das Verfügbare auch sinnvoll eingesetzt oder sind unsere Patienten überversorgt? Werden die Patienten nach den Prinzipien Evidenz-basierter Medizin behandelt? Sind die Ergebnisse der Untersuchungen und Interventionen so gut, wie sie sein könnten? Sind die tätigen Ärzte für ihre Eingriffe optimal ausgebildet und auch genügend erfahren? Kann sich das ärztliche Tun eines Zentrums mit den Besten messen? Gewiss, wir gehen davon aus, doch wirklich wissen wir es nicht: Zwar gibt es Leitlinien (inter)nationaler Gesellschaften, aber Qualitätsregister, wie sie die skandinavischen Länder [13] oder das United Kingdom [12] haben, sind in unserem Land von Ausnahmen abgesehen nicht verfügbar - und wegen der Kantonalisierung wohl kaum je zu haben. Das Register des «National Institute for Cardiovascular Outcomes Research» in England weist die Ergebnisse aller Eingriffe transparent bis hinunter auf die Zentren und Operateure aus. Anders als befürchtet, hat dies weder die Indikations- stellung noch die Ergebnisse beeinträchtigt - ganz im Gegenteil [13]. Des Weiteren zeigen diese Register, dass das Eingriffsvolumen medizinischer Zentren, nicht das der einzelnen Operateurs die Ergebnisse bestimmt - grosse Zentren liefern bessere Qualität. Zu viele kleine Zentren, wie sie der zerstückelte schweizerische Markt mit sich brachte, liegen auch medizinisch quer in der Landschaft.

\section{Ein "Conflict of Interest» der neuen Art}

Ein Weiteres gilt es zu beachten: Gibt es einen Schwellenwert der Konkurrenz, welcher vernünftige Medizin behindert? Gibt es wie in der Physiologie eine Dosis-AntwortKurve von Qualität und Konkurrenz, eine Frank-StarlingKurve von klinischem Ergebniss und finanziellem Druck? Könnte es sein, dass bei zu viel Konkurrenz die Kurve wie bei einem überfüllten Herzen wieder nach unten zeigt?

Ohne Zweifel gehört zur guten Ärztin/zum guten Arzt auch das Neinsagen, der Verzicht auf einen rentablen Eingriff von fraglichem Nutzen. Wenn der Kampf um Patienten sich zuspitzt, der Zustrom nicht dem Businessplan entspricht [14], entsteht ein «Conflict of Interest» der neuen Art. Wenn die Managerin/der Manager bei rückläufigen Zahlen der Ärztin/dem Arzt fordernd auf die Schulter klopft, kann sich dann Evidenz-basierte Medizin noch durchsetzen?

Die Überversorgung droht uns alle zu verführen: Der viel, zu viel behandelte Patient wiegt sich leicht in falscher Sicherheit, wähnt sich besonders umsorgt, glaubt das Beste zu erhalten; was er aber braucht, ist das Richtige und Nötige. Er ist sich weder der Kosten noch des Nutzens voll bewusst ist, denn er bezahlt nicht selbst. Der Patient als Kunde nutzt nur beschränkt den Wettbewerb, weil er es entweder wegen seiner Versicherung nicht kann oder die Unterschiede nicht kennt oder einsehen kann. Preisschwankungen nach Angebot und Nachfrage sind auch in der ökonomisierten Medizin nicht vorgesehen - also nur ein halber Markt!

\section{Unrentables}

Dann ist auch nicht alles, was die Medizin bietet, rentabel: Komplexe Fälle, alte Patienten, kontemplative Fächer, Ausund Weiterbildung, Forschung sind in der Welt des Gewinns nicht zu Hause. Selbst in akademischen Häusern kommt das Unrentable unter Druck. Allgemeinpatienten werden zur Belastung, unerfahrene Assistenten vermindern, wie man hört, die Effizienz des Betriebs; Paraprofessionals wären rentabler. Forschung droht zu einer Grille zu werden, die man, wenn überhaupt, ausserhalb der Arbeitszeit und abseits des Betriebs zu verrichten hat. Kurz: Das Budget wird zur Beurteilungsgrundlage von allem und jedem. 
Dabei ist letztlich den meisten klar: Die Assistenten sind die Ärzte von morgen, die Forschung die Medizin der Zukunft. Werden sie durch Budgets und kurzfristiges Renditedenken bedrängt, erstickt Innovation, die langfristig Gewinn erbringt. Schliesslich wollen auch unrentable Leiden behandelt werden, auch wenn sie heute im Marketing der Manager fehlen.

\section{The Bottom Line}

Kehren wir zur anfangs gestellten Frage zurück: Wenn wir wie damals Rousseau die Auswirkungen wirtschaftlichen Denkens auf die Medizin der heutigen Tage zu beurteilen hätten, was könnten wir sagen? Hat der Geist des Kapitalismus zur Veredelung oder zur Verderbnis der Sitten in der Medizin beigetragen? Wir wollen nicht eine «retour au passé», das wäre naiv und blind. Der Wettbewerb hat uns alle beflügelt und die Medizin verbessert.

Dennoch können wir das ungute Gefühl nicht verhehlen, dass die gute Ärztin/der gute Arzt in diesem Umfeld nicht wie früher gedeiht - Überversorgung, Public Relations, Reklame, ja der Kampf um Patienten haben die Lage überspannt. Gewiss, auch die Medizin kann sich heute der Frage ihrer Finanzierung nicht entziehen, insofern sind markwirtschaftliche Massnahmen zwingend und nützlich. Allerdings bleibt es eine halbe Sache: Der Markt ist durch die Kantonalisierung unnötig geschützt und durch unnötige Zentren verfälscht, die Preise sind vorgegeben und der Kunde weiss selten, was er kauft. Es bräuchte also wenigstens einen nationalen Markt, transparente Qualitätsausweise der Leistungen und des Eingriffsvolumens aller Zentren und eine stärkere finanzielle Beteiligung des Patienten, denn nur dann wird er zum Kunden (Abb. 2).

Zuletzt aber kann in einem Fach, in dem es auch um Zuwendung, um die Sorge um den Patienten und nicht bloss um das Rentable geht, marktwirtschaftliches Denken alleine nicht leitend sein. Ja, eine einseitige Ausrich- tung auf den Gewinn bedroht das Eigentliche unseres Fachs: Zuwendung für alle, Sorge um den Nachwuchs und Gestaltung unseres Wissens.

\section{Bibliografie}

1. Luther M: Bibel oder die ganze heilige Schrift des Alten und Neuen Testaments nach der deutschen Übersetzung. LukasEvangelium. Berlin, 1902; 10. Kapitel:Vers 30-35, S. 63.

2. Kant I: Kritik der praktischen Vernunft. Brandt HD, Klemme HF (Hrsg.). Hamburg; Felix Meiner Verlag: 2003.

3. Die Werke des Hippokrates. Die hippokratische Schriftensammlung in neuer deutscher Übersetzung. Kapferer R, Sticker G (Hrsg.), I-V, Stuttgart 1933-1940; Neuauflage: Hippokrates. Sämtliche Werke in 3 Bänden. Nach der Originalausgabe von 1933. Übersetzung: R. Kapferer. Anger; Anger-Verlag Eick; 1995.

4. Jenner $E$ : The three original publications on vaccination against smallpox. Harvard Classics Band 38. New York; Collier \& Son: 1910.

5. Lüscher TF: Das Arbeitsgesetz und der Geist der Medizin. Cardiovasc Med 2016; 19:165-169.

6. Bank I, Vliegen HW, Bruschke AV: The 200th anniversary of the stethoscope: Can this low-tech device survive in the hightech 21st century? Eur Heart J 2016; 37: 3536-3543.

7. Lüscher TF, Obeid S: From Eisenhower's heart attack to modern management: a true success story! Eur Heart J 2017; 38: 3066-3069.

8. Meier B, Bachmann D, Lüscher TF: 25 years of coronary angioplasty: almost a fairy tale. Lancet 2003; 361: 527.

9. Lüscher TF: Ist die Medizin ein Business? Cardiovasc Med $2007 ; 7: 351-355$

10. Lüscher TF: Zahlenmystik rund ums Herz - und was daraus zu lernen wäre. Cardiovasc Med 2010; 13: 357-336.

11. Rousseau JJ: Abhandlung über die von der Akademie zu Dijon gestellte Frage, ob die Wiederherstellung der Wissenschaften und Künste zur Läuterung der Sitten beigetragen habe. In: Sozialphilosophische und politische Schriften. Düsseldorf/ Zürich; Artemis Winkler; 1996. S. 11.

12. Lüscher TF, Obeid S: From Eisenhower's heart attack to modern management: a true success story! Eur Heart J 2017; 38: 3066-3069.

13. Szummer K, Wallentin L, Lindhagen L, et al.: Improved outcomes in patients with ST-elevation myocardial infarction during the last 20 years are related to implementation of evi-

\begin{tabular}{|c|c|}
\hline Strength & Weakness \\
\hline $\begin{array}{l}\text { - Device- und Instru- } \\
\text { mentenentwicklung } \\
\text { - Medikamentenent- } \\
\text { wicklung } \\
\text { - Klinische Innovation } \\
\text { - Service } \\
\text { - Effizienz } \\
\text { - Kundenorientierung } \\
\text { - Oekonomische } \\
\text { Realität }\end{array}$ & $\begin{array}{l}\text { - Segmentierter Markt } \\
\text { (Kantonalisierung) } \\
\text { - Fixe Preise } \\
\text { - Geringe } \\
\text { Kundenbeteiligung } \\
\text { - Keine Beziehung } \\
\text { Kosten - Qualität } \\
\text { - Wenig Qualitäts- } \\
\text { transparenz } \\
\text { - Kaum Outcomes } \\
\text { Research }\end{array}$ \\
\hline
\end{tabular}

\begin{tabular}{l} 
Opportunities \\
\hline - Landesweite Markt- \\
ausweitung \\
- Outcomes Register \\
Patienten- und Ein- \\
griffsvolumen Re- \\
porting \\
- Kostentransparenz \\
Overhead für Lehre \\
und Forschung \\
\end{tabular}

\section{Threats}

- Fokussierung auf rentable Leiden

- Profitdruck auf Aerzte und Pflege

- Paraprofessionals anstatt Assistenten

- Verdrängung der Forschung

- Beeinträchtigung Weiterbildung

Abbildung 2. SWOT Analyse der Ökonomisierung der Medizin. 
dence-based treatments: experiences from the SWEDEHEART registry 1995-2014. Eur Heart J 2017; 38: 3056-3065.

14. Hulme W, Sperrin M, Curzen N, et al.: British Cardiovascular Intervention Society and the National Institute of Cardiovascular Outcomes Research. Operator volume is not associated with mortality following percutaneous coronary intervention: insights from the British Cardiovascular Intervention Society registry. Eur Heart J 2018; 39: 1623-1634.

15. Jones DA, Krishnaraj S, Rathod, Sudheer K, et al.: The association between the public reporting of individual operator outcomes with patient profiles, procedural management, and mortality after percutaneous coronary intervention: an observational study from the Pan-London PCI (BCIS) Registry using an interrupted time series analysis. Eur Heart J 2019; 40: 2620-2629.
16. Fey MF: Privatklinikkgruppe Hirslanden. Jahresbericht 2006 ein paar kritische Kommentare. Schweiz Arzte Ztg 2007; 88: $1309-1311$

\section{Prof. Dr. med. Thomas Lüscher, FESC, FACC, FRCP}

Professor of Cardiology at Imperial College

Director of Research, Education \& Development

Royal Brompton \& Harefield Hospitals and

National Heart and Lung Institute

London, SW3 6NP, United Kingdom

cardio@tomluescher.ch 